\title{
Dental amalgam implantation and thyroid autoimmunity
}

\author{
Kisakol G \\ Acibadem University Medical Faculty, Acibadem Bursa Hospital, Turkey.gurcank@hotmail.com
}

\begin{abstract}
Objective: Mercury was heavily studied as a factor in the autoimmune processes. We aimed to observe whether mercury of amalgam is associated with Hashimoto disease.

Background: 363 patients with Hashimoto's thyroiditis and 365 control subjects were included in to the study. Amalgam fillings were checked by the physician.

Methods: 363 (49.9 \%) patients and 365 (50.1\%) healthy controls were included into the study. Hashimoto's thyroiditis was diagnosed with thyroid hormones, antithyroid antibody levels, and ultrasonographic findings. Control subjects were selected from patients with no known autoimmune diseases. They were controlled with ultrasonography, as well as antibody titers. None of them had Hashimoto's thyroiditis.

Results: Sex distribution of the study population was following: $319(87.9 \%)$ female, $44(12.1 \%)$ male in the patient group and $277(75.9 \%)$ male and $88(24.1 \%)$ female in healthy control subjects, respectively. Mean free T4 values for Hashimoto's thyroiditis and healthy control group were $15.30 \pm 0.76,17.30 \pm 0.96 \mathrm{pmol} / \mathrm{L}$ and mean TSH values for Hashimoto's thyroiditis and healthy control group were $9.29 \pm 20.79,1.20 \pm 0.32 \mathrm{ulU} / \mathrm{ml}$. Frequency of dental amalgam implantation in patients with Hashimoto's thyroiditis was not statistically significantly different from healthy controls $(p=186)(t=-1.324)$

Conclusions: Some studies identified mercury of amalgam as responsible for autoimmune thyroiditis. We studied whether amalgam fillings are more frequent in Hashimoto's thyroiditis patients and whether it is a causative factor for Hashimoto's thyroiditis. Statistical analysis revealed that there is no relation of amalgam with Hashimoto's thyroiditis (Tab. 1, Ref. 34). Text in PDF www.elis.sk.

Key words: Hashimoto's thyroiditis; thyroiditis; autoimmune; amalgam; mercury.
\end{abstract}

The human body is constantly exposed to metals and metal containing compounds. Some are crucial in trace amouts for normal metabolic function, but some are toxic. The key factor for toxicity is time of exposure, cumulative concentration and genetic susceptibility (1). Dental amalgam filling which contains approximatelly $50 \%$ of mercury is accepted a major source of mercury exposure in general population $(2,3)$. Studies performed with sheep and monkey have shown that substantial amount of amalgam released from insertion of dental amalgam fillings accumulates in the body $(4,5)$. It is estimated that 100 million of amalgam fillings are inserted annually in USA (6). Immune system is of special interest as a target for low dose inorganic mercury (3). Case reports of accidental mercury exposure have shown that mercury can induce autoimmune reactions and/or systemic immune deposits in some individuals especially prone to this $(7,8,9)$. In rodent models it was clearly shown that mercury induced systemic autoimmunity is strongly dependent on genetic constitution (10). Studies in animal models susceptible to mercury proposed that potential mechanisms by which inorganic mercury induced autoimmunity are $\mathrm{T}$ cell dependent activation and induction of autoimmune conditions, implying lymphoproliferation, immunostimulation of antinuclear antibody and immune complex formation (11). Besides mercury, implantation of alloy contain-

Acibadem University Medical Faculty, Acibadem Bursa Hospital, Turkey Address for correspondence: Kisakol G, Acibadem Bursa Hospital, Acibadem University Medical Faculty, Sumer Sk. No: 1 Nilufer, Bursa, Turkey. Phone: +905422629638, Fax: +902244532201 ing silver as well as oral silver exposure induce autoimmunity in genetically sensitive mouse strains $(12,13,14)$. Endocrine organs can be a target for an autoimmune attack, leading to organ specific autoimmune disease such as thyroid autoimmunity (15). For development of thyroid autoimmunity several factors are required; These are genetic predisposition linked mainly to antigens of HLA system, namely HLA B8, B15, B5, DR3, DR4 and enviromental factors such as viral and bacterial factors, iodine intake and heavy metal exposure.

While many researchers claimed that amalgam fillings contribute to a series of illnesses, many others did not identify such association. Latter group states that most people with fillings have less than 5 micrograms per liter of urine. Nearly all practicing dentists have levels below 10 micrograms per liter, even though they are exposed to mercury vapor when placing or removing amalgam filings and typically have amalgams in their own teeth. Thus, even with that exposure, the maximum levels found in dentists are only slightly higher than those of their patients and are far below the levels known to effect health, even in a minor way (16).

In this study, we intended to determine any relation between dental amalgam implantation and autoimmunity in patients with Hashimoto's thyroiditis.

Tab. 1. Statistical analysis of dental amalgam fillings between thyroiditis patients and control subjects.

\begin{tabular}{lccc}
\hline & $\begin{array}{c}\text { Dental } \\
\text { amalgam (+) }\end{array}$ & $\begin{array}{c}\text { Dental } \\
\text { amalgam (-) }\end{array}$ & p value \\
\hline Hashimoto's thyroiditis & $20055.7 \%$ & $15944.3 \%$ & $>0.05$ \\
Control & $19052.1 \%$ & $17547.9 \%$ & \\
\hline
\end{tabular}




\section{Subjects and methods}

363 patients with Hashimoto's thyroiditis diagnosed in Endocrinology department of Acibadem Bursa Hospital according to thyroid hormone levels, antimicrosomal, antitiroglobulin antibody serum levels, clinical and ultrasonography findings. 365 control subjects were included into the study. Control subjects were selected from patients admitted to hospital with nodular goiter diagnosis. They were controlled with ultrasonography, as well as antibody titers. None had Hashimoto's thyroiditis. Those with type 1 diabetes mellitus, pernicious anemia, Addison disease, autoimmune hepatitis, autoimune rheumatological and autoimmune hematological diseases, chronic liver disease, chronic renal disease, malignancy, viral hepatitis, pregnancy were excluded from the study. Also patients who were treated with interleukine, interferon and amiodarone were excluded from the study. Cases over 18 years old were included into the study. Patients were examined by endocrinologists, and amalgam fillings were noted. The study was performed with the permission of local ethical committee. For non-parametric analysis chi-square test was used. Anova was used for multiple comparisons between blood group antigens. $\mathrm{p}<0.05$ was accepted statistically significant.

\section{Results}

$363(49.9 \%)$ patients and $365(50.1 \%)$ healthy controls were included into the study. Sex distribution was following: $319(87.9 \%)$ female, $44(12.1 \%)$ male in patient group and $277(75.9 \%)$ male and $88(24.1 \%)$ female in healthy control subjects, respectively. Mean free T4 values for Hashimoto's thyroiditis and healthy control group were $15.30 \pm 0.76,17.30 \pm 0.96 \mathrm{pmol} / \mathrm{L}$ and mean TSH values for Hashimoto's thyroiditis and healthy control group were 9.29 \pm 20.79 , $1.20 \pm 0.32 \mathrm{uIU} / \mathrm{ml}$. Frequency of dental amalgam implantation in patients with Hashimoto's thyroiditis is not statistically significantly different from healthy controls $(\mathrm{p}=186)(\mathrm{t}=-1.324)$ (Tab. 1). According to risk analysis (OR: 0.863) $95 \%$ confidence interval (0.644-1.156) there is no high risk attributable to amalgam fillings.

\section{Discussion}

Hashimoto's thyroiditis is the most frequent organ specific autoimmune disorder (15). Infiltrating lymphocytes and T cell mediated cytotoxicity are involved in the pathogenesis of autoimmune thyroiditis (16). It is seen more commonly in females. Gender, iodine insufficiency, smoking and aging are known risk factors related with Hashimoto's thyroiditis.

Metals in general, and mercury in particular have long been known as immunosupressive agents. Although mercury is known as immunosupressant, it was also shown to be stimulant to cellular and humoral immune system and even was regarded as responsible for autoimmunity (13). Many immunological effects caused by implantation of the amalgam such as hyperimmunglobulinemia, development of serum autoantibodies with a nucleolar pattern/ anti fibrillarin antibodies (ANoA/ AFA), potentiation of mitogen induced lymphocyte proliferation and increased expression of class II molecules are consistent with the known effects of mercuric chloride on immune system in genetically susceptible mice and rats $(17,18,19)$. Alloy/amalgam implantation or directly amalgam including mercuric chloride in SJL mice has precipitated the presence of serum autoantibodies with a nucleolar pattern (ANoA) against fibrillarin (20). Antifibrillarin antibodies are of interest in the medicine due to in nearly half of the scleroderma patients, ANoA targetting the fibrillarin was detected (21). ANoa/ AFA have been elucidated from kidneys from mercury treated SJL mice with immune complex disease indicating pathogenetic role of autoantibodies in this pathology (22). On the other hand long term exposure to mercury from dental amalgam has not been shown to have causative role in the production of anti-glomerular basement membrane antibodies (23).

The reduction of mercury specific response following amalgam removal has been published in patients with thyroid autoimmunity (24). Amalgam mercury significantly contributes to long term thyroid burden of mercury (25). Kawada et al showed $50 \%$ reduction in Na-K-ATPase activity in the membranous preperations from dog thyroid gland by mercuric chloride in $10-{ }^{7} \mathrm{M}$ concentrations (26). Following intraperitoneal injection of mercury de novo sysnthesis of iodothyronins was significantly decreased in mice. This suggests mercury may cause coupling defect in the synthesis of iodothyronins. In male Brown Norway rats $\mathrm{HgCl}_{2}$ leads to production of autoantibodies capable of binding thyroglobulin (27). Prochazkova, Sterzl et al reported that lymphocyte reactivity measured with MELISA indicating in vitro reactivity after the replacement of dental amalgam decreased significantly to inorganic mercury, silver, organic mercury and lead. (28)

According to WHO Environmental Health Criteria, the estimated average release of elemental mercury vapour from dental amalgams in the general population is between 3.8-21 jig/day, and its retention in the body is $3-17 \mathrm{jg} /$ day. According to another FAO/WHO report, the maximum weekly mercury intake with food regarded as having no adverse effect on health, is $300 \mathrm{pg}$ in the form of inorganic mercury compounds and 200 pg per week in the form of methyl mercury (29).

According to National Council Against Health Fraud position paper on amalgam filling there is no reason to remove amalgam fillings other than amalgam induced allergy and problems related with fillings (30). In a controlled study, it was shown that many symptoms atrributed to amalgam mercury were alleviated both by placebo and mercury chelation therapy, so there is currently not enough evidence to support for the recommendation for removal of amalgam in autoimmune disorders (31).

With respect to the reduced lymphocyte responses, Mackert et al (32) showed no indication that amalgam affects the human immune system, and no association was found between the number of amalgam fillings in individuals and Henoch-Schönlein purpura and acute glomerulonephritis, which are all autoimmune diseases. Epidemiologic studies have shown that occupational exposure to mercury does not usually result in autoimmunity. Mercury does induce antinuclear antibodies, scleroderma-like diseases, lichen planus, or membranous nephropathy in some individuals, however, immunogenetic and pharmacogenetic factors are responsible 
for the induction of metal-associated autoimmunity in general (33). In addition to estrogen replacement therapy, other factors including mercuric chloride are putative risk factors for the development of lupus, scleroderma, and Raynaud disease. Urinary mercury excretion has been reported to be higher in scleroderma patients who are positive for antifibrillarin antibodies. However this level is still in the normal and unexposed ranges, and these patients never develop immune complex glomerulonephritis (34).

In our study, we did not observe any significant relation between amalgam and autoimmune thyroiditis, nor there was increased risk. Non-evaluation of fecal and urine silver and mercury levels that represents the release of these metals from amalgam implants was the limitation of present study however as mentioned previously urine excretion of mercury in amalgam patients is not over normal limits. In the light of some studies it may be assumed that mercury of amalgam might be responsible for autoimmune diseases. We did not study other possible side effects of mercury over immune system however according to our results mercury is not responsible for thyroid autoimmunity.

\section{References}

1. Shitova O, Guseva L, Denisova A. Immunosupression caused by industrial chemicals in workers of a pharmaceutical factory. In: 8th International Congress of lmmunology; August, 1992; Budapest, Hungary: Springer Hungarica, p. 597.

2. Clarkson, T W, Friberg L, Hursh JB, Nylander M. The prediction of intake of mercury vapor from amalgams. In: Clarkson, TW, Friberg L, Nordberg GF, Sager PR (Eds). Biological Monitoring of Toxic Metals. 247-264. New York; Plenum, 1988.

3. WHO. Environmental Health Criteria 118. Inorganic mercury IPCS. Geneva: World Health Organization, 1992.

4. Hahn LJ, Kloiber R, Leininger RW, Vimy MJ, Lorscheider FL. Dental 'silver" tooth fillings: a source of mercury exposure revealed by whole-body image scanning and tissue analysis. FASEB J 1989; (3): 2641-2646.

5. Hahn LJ, Kloiber R, Leininger RW, Vimy MJ, Lorscheider FL. Wholebody imaging of the distribution of mercury released from dental amalgam fillings into monkey tissues. FASEB J 1990; (4): 3256-3260.

6. When your patients ask about mercury in amalgam. J Am Dent Assoc 1989; 120: 395-398.

7. Roger J, Zillikens D, Burg G, Gleichmann E. Systemic autoimmunity in a patient with long standing exposure of mercury. Eur J Dermatol 1992; 2: $168-170$

8. Barr RD. The mercurial nephrotic syndrome. East Afr Med J 1990; 67: 381-386.

9. Schrallhammer-Benkler K, Ring J, Przybilla B, Meurer M, Landthaler M. Acute mercury intoxication with lichenoid drug eruption followed by mercury contact allergy and development of antinuclear antibodies. Ada Dermato-Venereol 1992; 72: 294-302.

10. Bigazzi PE. Lessons from animal models: the scope of mercury induced autoimmunity. Clin Immunol Immunopathol 1992; 65: 81-84.

11. Martinsson K, Hultman $P$. The role of Fc-receptors in murine mercury-induced systemic autoimmunity. Clin Exp Immunol 2006; 44: 309-318.

12. Hultman P, Enestrom S, Turley SJ, Pollard KM. Selective induction of antifibrillarin autoantibodies by silver nitrate. Clin Exp Immunol 1994; 96: 285-291.

13. Hultman P, Johansson U, Turley SJ, Lindh U, Enestrom S, Pollard KM. Adverse immunological effects and autoimmunity induced by dental amalgam and alloy in mice. FASEB J 1994; 8 (14): 1183-1190.
14. Hultman P, Ganowiak K, Turely SJ, Pollard KM. Genetic susceptibility to silver-induced antifibrillarin autoantibodies in mice. Clin Immunol Immunopathol 1995; 77: 291-297.

15. Sterzl I, Prochazkova J, Hrda P, Matucha P, Bartova J, Stejskal V. Removal of dental amalgam decreases anti-tpo and anti-tg autoantibodies in patienst with autoimmune thyroiditis. Neuroendocrine lett 2006; 27 (Suppl 1): 25-30.

16. Stassi G, De Maria R. Autoimmune thyroid disease: new models of cell death in autoimmunity. Nat Rev lmmunol 2002; 2 (3): 195-204.

17. Hultman P, Johansson U. Strain differences in the effect of mercury on murine cell-mediated immune reactions. Food Chem Toxicol 1991; 29: 633-638.

18. Dubey C, Bellon B, Hirsch F, Kuhn J, Vial MC, Goldman M, Druet P. Increased expression of class II major histocompatibility complex molecules on $\mathrm{B}$ cells in rats susceptible or resistant to $\mathrm{HgCI}$-induced autoimmunity. Clin Exp Immunol 1991; 86: 118-123.

19. Hultman P, Eneström S. The induction of immune complex deposits in mice by peroral and parenteral administration of mercuric chloride: strain dependent susceptibility. Clin Exp Immunol 1987; 67: 283-292.

20. Turley SJ, Tan EM, Pollard KM. Molecular cloning and sequence analysisof U3 snoRNA associated mouse fibrillarin. Biochem Biophys Acta 1216 1993; (1): 119-122.

21. Reimer G, Steen VD, Penninger CA, Medsger TA, Tan EM. Correlates between autoantibodies to nucleolar antigens and clinical features in patients with systemic sclerosis (scleroderma). Arthritis Rheum 1988; 31: 525-532.

22. Hultman P, Eneström S. Mercury induced antinuclear antibodies in mice: characterization and correlation with renal immune complex deposits. Clin Exp Immunol 1988; 71 (2): 269-274.

23. Guzzi G, Fogazzi GB, Cantù M, Minoia C, Ronchi A, Pigatto PD. Dental amalgam, Mercury toxicity, and renal autoimmunity. J Environ Pathol Toxicol Oncol 2008; 27 (2): 147-155.

24. Sterzl I, Procházková J, Hrdá P, Bártová J, Matucha P, Stejskal VD. Mercury and nickel allergy: risk factors in fatigue and autoimmunity. Neuroendocrinol Lett 1999; 20: 221-228.

25. Guzzi G, Grandi M, Cattaneo C, Calza S, Minoia C, Ronchi A. Dental amalgam and mercury levels in autopsy tissues: food for thought. Am J Forensic Med Pathol 2006; 27 (1): 42-45.

26. Kawada J, Nishida M, Yoshimura Y. Effects of organic and inorganic mercurials on thyroidal functions. Pharmacobiodyn 1980; 3 (3): 149-159.

27. Pusey CD, Bowman C, Morgan A, Weetman AP, Hartley B, Lockwood CM. Kinetics and pathogenecity of autoantibodies induced by mercuric chloride in the Brown Norway rat. Clin Exp Immunol 1990; 81:7 6-82.

28. Prochazkova J, Sterzl I, Kucerova H, Bartova J, Stejskal VD. The beneficial effect of amalgam replacement on health in patients with autoimmunity. Neuro Endocrinol Lett 2004; 25: 211-218.

29. Stadtler P. Dental amalgam III: toxicity. Int J Clin Pharmacol Therap Toxicol 1991; 29: 168-171.

30. National Council Against Health Fraud. Position paper on amalgam fillings, 2002.

31. Grandjean P, Guldager B, Larsen IB, Jørgensen PJ, Holmstrup. Placebo response in enviromental disease: Chelation therapy of patients with symptoms attributed to amalgam fillings. J Occup Environ Med 1997; 39: 707-714 .

32. Mackert JR, Leffel MS, Wagner DA, Powell BJ. Lymphocyte levels in subjects with and without amalgam restorations. JADA 1991; 122 (3): 49-53.

33. Bigazzi PE. Metal and kidney autoimmunity. Environ Health Perspect 1999; 107 (Suppl 5): 753-765.

34. Mayes MD. Epidemiological studies of environmental agents and systemic autoimmune diseases. Environ Health Perspect 1999; 107 (Suppl 5): 743-748.

Received February 27, 2012. Accepted August 18, 2013. 